Published Ahead of Print on September 4, 2008 as 10.1097/PSY.0b013e318183a491

\title{
Individual and Neighborhood Socioeconomic Status Characteristics and Prevalence of Metabolic Syndrome: The Atherosclerosis Risk in Communities (ARIC) Study
}

\author{
Kristal L. Chichlowska, MPH, Kathryn M. Rose, PhD, Ana V. Diez-Roux, MD, PhD, MPH,
} Sherita H. Golden, MD, MHS, Annie M. McNeill, PhD, and Gerardo Heiss, MD, PhD

\begin{abstract}
Objective: The objective of this study was to examine the association of individual socioeconomic status (iSES) and neighborhood SES (nSES) on the prevalence of metabolic syndrome (MetS) in the Atherosclerosis Risk in Communities Study (1987-1999). Methods: Participants included 2932 black and 9777 white men and women aged 45 to 64 years without diabetes at baseline. Total combined family income for the past 12 months and six census tract socioeconomic measures combined into a composite index were used to quantify iSES and nSES, respectively. Poisson regression was used to assess associations of the joint contribution of iSES and nSES on the MetS, stratified by gender and race and adjusting for multiple covariates. For analyses that included nSES, hierarchical modeling techniques were used. Results: Using 2005 Third Report of the Expert Panel on Detection, Evaluation, and Treatment of High Blood Cholesterol in Adults criteria, MetS was identified in 40\% of black women, 30\% of white women, $28 \%$ of black men, and 35\% of white men. Among black and white men, there was no association between MetS and iSES or nSES. In contrast, after adjustment for risk factors, black and white women with low (L)-iSES and medium (M)-iSES were more likely to have MetS than those with high (H)-iSES. Similar but weaker patterns were noted for L-nSES and M-nSES. Conclusions: In summary, both iSES and nSES were independently associated with an increased prevalence of MetS among women but not men. Efforts aimed at understanding the causes of these gender differences may offer insight into avenues for reducing the prevalence of the MetS and its chronic disease sequelae. Key words: SES, income, neighborhood, metabolic syndrome, syndrome X, insulin resistance, stress.
\end{abstract}

MetS = metabolic syndrome; CVD = cardiovascular disease; SES = socioeconomic status; iSES = individual-level socioeconomic status; nSES = neighborhood-level socioeconomic status; ARIC $=$ atherosclerosis risk in communities study; $\mathbf{N C}=$ North Carolina; MS = Mississippi; MN = Minnesota; $\mathbf{M D}=$ Maryland; $\mathbf{L}=$ low $\mathbf{M}=$ middle; $\mathbf{H}=$ high; $\mathbf{L D L}-\mathbf{C}=$ low-density lipoprotein cholesterol; $\mathbf{P R}=$ prevalence ratio; $\mathbf{C I}=$ confidence interval.

\section{INTRODUCTION}

$\mathrm{T}$ he metabolic syndrome (MetS) has been identified as a cluster of cardiovascular disease (CVD) risk factors that includes defects in insulin sensitivity (impaired fasting glucose), dyslipidemia, hypertension, and central adiposity $(1,2)$. Its prevalence in a cross-sectional (1988-1994) nationally representative sample of US adults was almost $25 \%$ and differed by age, race, and gender (3). MetS is associated with increased risk of diabetes $(4,5)$, atherosclerosis (6), coronary heart disease (6-8), and CVD $(5,7-9)$ morbidity and mortality. Consistent inverse associations between socioeconomic status (SES) and some components of the MetS, such as large waist (10-13), defects in insulin sensitivity (13-16), and high blood pressure $(13,17,18)$ have been reported in the literature,

From the Department of Epidemiology (K.L.C., K.M.R., G.H.), School of Public Health, the University of North Carolina at Chapel Hill, North Carolina; Department of Epidemiology (A.V.D.-R.), University of Michigan School of Public Health, Ann Arbor, Michigan; Department of Medicine (S.H.G.), Johns Hopkins University School of Medicine, Baltimore, Maryland; and Merck and Co. (A.M.M.), North Wales, Pennsylvania.

Address correspondence and reprint requests to Kristal Chichlowska, MPH, Bank of America Center, 137 E Franklin St, Ste 306, Chapel Hill, NC 27514. E-mail: raymon@email.unc.edu

This research was supported by the National Heart, Lung and Blood Institute Grant No. 1R01HL080287 (to K.M.R.) and 1R01HL080287 to 01S1 (to K.M.R, K.L.C.). The Atherosclerosis Risk in Communities Study is carried out as a collaborative study supported by contracts from the National Heart, Lung, and Blood Institute (N01-55015, N01-55016, N01-55018, N01-55019, N01-55020, N01-55021, N01-55022, and R01-HL064142).

Received for publication December 19, 2007; revision received June 5, 2008 DOI: 10.1097/PSY.0b013e318183a491 although the association between SES and dyslipidemia $(19,20)$ is less consistent. Furthermore, US (21-26) and non-US studies (27-36), suggest an association between individual-level SES (iSES) indicators, such as income, education or occupational status, and the MetS in adults. Among these studies that conducted gender-specific analyses, many found the association to be greater among women than men (21-23,26-31,33,34), whereas others found comparable associations between gender groups $(25,35)$.

Studies $(13,37-40)$ have also shown that living in lower SES neighborhoods is associated with higher levels of CVD and its related risk factors, with effects, while attenuated, that generally persisted after considering iSES. The processes through which neighborhood characteristics could independently affect MetS risk are probably similar to those hypothesized for CVD, coronary heart disease, and their risk factors. It has been suggested that neighborhood-level SES (nSES) indicators, not captured by individual-level measures, affect health through their association with neighborhood characteristics. Neighborhood characteristics include material infrastructures and services that affect social norms that influence health behaviors $(13,41)$, psychosocial health $(42,43)$, access to healthy foods (44), and opportunities for safe recreation (43). To our knowledge, only one study has considered the impact of neighborhood socioeconomic conditions on the cluster of components of the MetS in young adults (38), and results differed by race and gender.

iSES influences the choice of neighborhood where one resides. Likewise, neighborhood socioeconomic conditions influence the educational and occupational opportunities of their residents and have been shown to be differentially distributed in iSES categories (45). Thus, iSES may be conceptualized as both a mediator and a confounder of the relationship between nSES and health. Furthermore, nSES may operate as a confounder or be on the pathway between iSES and health. To disentangle their relationships, it is important to evaluate 
the independent effects of both iSES and nSES on the MetS. We examined the cross-sectional associations between iSES and nSES in a middle-aged black and white cohort of men and women, the Atherosclerosis Risk in Communities (ARIC) Study. We hypothesized that both iSES and nSES would be inversely associated with MetS. Given that in the United States, socioeconomic and neighborhood characteristics tend to differ among black and white persons, and because the prevalence of the MetS differs by race and gender, we examined whether SES-MetS associations, if present, varied by race and gender.

\section{METHODS}

\section{Study Population}

The ARIC study is a community-based prospective cohort designed to investigate the etiology and natural history of atherosclerosis and its sequelae. From 1987 to 1989 , the ARIC study examined 15,792 black and white residents aged 45 to 64 years in four communities in North Carolina (NC), Mississippi (MS), Minnesota (MN), and Maryland (MD). Two of the samples (Washington County, MD and Minneapolis, MN suburbs) were mostly white. Blacks were over-sampled in Forsyth County, NC (12\% black) and were exclusively sampled in Jackson, MS to provide sufficient power to investigate findings by ethnicity. A comparison of participants with nonrespondents has been previously described (46). The institutional review boards at the involved institutions approved the study, and all participants gave informed, written consent. A detailed description of the ARIC study has been published (47).

We excluded participants with diabetes $(\mathrm{n}=1870)$ or an unknown diabetes status at baseline $(n=122)$, with missing data that precluded classification of the MetS $(n=885)$ and $\operatorname{nSES}(n=663)$, with a race other than black or white $(n=48)$ and black participants in Minneapolis, MN or Washington County, MD $(n=55)$. Type II diabetes was defined as a fasting blood glucose level greater than $140 \mathrm{mg} / \mathrm{dl}$, nonfasting blood glucose level greater than $200 \mathrm{mg} / \mathrm{dL}$, use of hypoglycemic medications or self-reported physician diagnosis. The final sample size was 12,709 .

\section{Ascertainment of SES}

The iSES exposures considered were family income and educational attainment. Educational attainment was categorized as less than high school, high school graduate or equivalent, and greater than high school. Family income, originally grouped into 10 ordinal categories, was collapsed into three categories that closely approximated tertiles (under \$24,000 (L-iSES), $\$ 24,000$ to $\$ 49,999$ (M-iSES), and $\$ 50,000$ or more (H-iSES)).

A commercial vendor geocoded place of residence at the baseline exam (1987-1989) and these data were used to link participants with 1990 US Census tract level socioeconomic data. Census tract level matches were obtained for $90 \%$ of the addresses submitted (48). The repeatability and accuracy of the vendor have been reported elsewhere and demonstrated to be high (49).

To provide comparability with earlier analyses of the neighborhood context in the ARIC study we used a composite index Z-score based on methods used by Diez-Roux et al. in earlier work (41,50). Six 1990 US census-based area measures were chosen to represent dimensions of wealth and income based on factor analyses: $\log$ of the median household income in 1989, $\log$ of the median value of owner occupied house, percent of households receiving interest, dividend, or net rental income, percent of adults $25+$ with high school degree, percent of adults $25+$ with a college degree, and percent of individuals aged $16+$ in professional, managerial, or executive occupations. For each measure chosen, a Z-score was calculated by subtracting the mean value of all tracts within the study communities and dividing by the SD. A neighborhood summary score was constructed by summing Z-scores for each of the six variables. The average values for all census tracts within the study communities were used as the mean. Because of limited overlap in black and white nSES, race-specific tertiles of summary scores were created.

\section{Metabolic Syndrome}

Blood pressure, triglycerides, high-density lipoprotein cholesterol, glucose, and waist-to-hip ratio were measured according to standard ARIC procedures (36). Participants were asked to fast 12 hours before blood draw and actual fasting times were recorded. Blood was drawn from an antecubital vein of seated participants, serum was centrifuged, and frozen samples were shipped to central laboratories for analysis. Trained technicians measured waist and hip circumferences to the nearest centimeter at the umbilical level and maximum buttocks, respectively. The mean of the last two of three systolic and diastolic sitting blood pressure measurements obtained from a random-zero sphygmomanometer was used for measures of blood pressure.

The MetS was defined as a binary outcome using the Third Report of the Expert Panel on Detection, Evaluation, and Treatment of High Blood Cholesterol in Adults criteria (1) as the presence of three or more of the following components: elevated blood pressure (systolic blood pressure $>130$ or diastolic blood pressure $>85 \mathrm{~mm} \mathrm{Hg}$ and/or use of antihypertension medications (yes/no)); elevated triglycerides ( $\geq 150 \mathrm{mg} / \mathrm{dl}$ ); low high-density lipoprotein cholesterol (men $<40 \mathrm{mg} / \mathrm{dl}$, women $<50 \mathrm{mg} / \mathrm{dl}$ ); impaired fasting (fasting $>8$ hours) glucose $(>100 \mathrm{mg} / \mathrm{dl})$; and large waist circumference (men $>102$ $\mathrm{cm}(>40$ in), women $>88 \mathrm{~cm}(>35 \mathrm{in})$.

\section{Covariates}

The covariates selected for the analysis were age (years), gender (male or female), smoking status and alcohol use (current, former, never), physical activity, low-density lipoprotein cholesterol (LDL-C in mg/dL), study center (Forsyth County, NC, Jackson, MS, Minneapolis, MN, and Washington County, MD) and self-reported race (black or white). Each participant was asked to indicate whether they were white, black, Indian, or Asian. "Race" refers to a socially constructed category and has been shown to correlate well with measured and unmeasured environmental attributes such as diet, occupational exposures, and social resources (51). Physical activity was measured using the sport during leisure time activity index (range, 1-5) of Baecke's questionnaire (52). LDL-C level was calculated by using the Friedewald formula (36) at a central laboratory.

\section{Statistical Analysis}

All analyses were performed with SAS 9.1 (SAS Institute, Inc., Cary, NC) and were gender and race specific. Characteristics of the population (Table 1) were described as proportions and means and the prevalence of the MetS (Figure 1) was described as age-adjusted proportions. The strength of associations between various measures for iSES and nSES and MetS (Table 3) were estimated by prevalence ratios (PR) and 95\% confidence intervals (CI) between low and medium SES and the high SES referent group.

Given the high prevalence of the MetS (greater than $10 \%$ in all race gender groups), Poisson regression, implemented using generalized estimation equations, was used to assess associations between SES and MetS as it has been shown to provide more accurate estimates of PRs in such circumstances (53). To prevent underestimation of standard errors owing to overdispersion, the Pearson scale option was used (54). Because analyses included nSES, hierarchical modeling techniques were used, as they take into account the dependence of persons from the same area in the calculation of the standard errors $(55,56)$. Included participants resided in 351 different census tracts, with a median number of 14 participants per tract. Interactions between gender, race, and nSES and iSES tertiles were tested through the Wald test comparing the models with and without interactions. All tests for interactions use $\alpha=0.05$ to determine statistical significance.

Analyses of associations between SES and the MetS were adjusted for age, smoking status, alcohol use, physical activity, LDL-C, and study center. The center and age variables were retained in the model, because they were found to be confounders of the SES-MetS relationship. Physical activity, LDL-C, smoking, and alcohol use were not found to be confounders. However, because they are regarded as strong risk factors for MetS in the literature, and did not change our point estimates, these variables remained in the model.

Further, in order to evaluate the independent effect of nSES on the MetS, we also controlled for iSES. Likewise, models for iSES controlled for nSES. 
SES AND THE METABOLIC SYNDROME

TABLE 1. Selected Baseline Characteristics of Included Participants by Race and Gender, Atherosclerosis Risk in Communities (ARIC) Study (1987-1989)

\begin{tabular}{|c|c|c|c|c|}
\hline $\begin{array}{c}\text { Characteristic } \\
n\end{array}$ & $\begin{array}{c}\text { Black Women } \\
1803\end{array}$ & $\begin{array}{l}\text { White Women } \\
\quad 5244\end{array}$ & $\begin{array}{c}\text { Black Men } \\
1129\end{array}$ & $\begin{array}{c}\text { White Men } \\
4533\end{array}$ \\
\hline $\begin{array}{l}\text { Age, mean } \pm \text { SD, yr } \\
\text { Neighborhood Z-Score (\%) }\end{array}$ & $53 \pm 5.7$ & $54 \pm 5.7$ & $54 \pm 6.0$ & $55 \pm 5.7$ \\
\hline Low & 36.2 & 31.7 & 38.0 & 33.0 \\
\hline Medium & 23.4 & 36.1 & 24.1 & 34.9 \\
\hline High & 40.4 & 32.2 & 37.9 & 32.1 \\
\hline \multicolumn{5}{|l|}{ Family income $(\%)^{b}$} \\
\hline$<\$ 25,000$ & 64.5 & 27.5 & 45.0 & 18.3 \\
\hline$\$ 25,000-\$ 49,999$ & 23.7 & 38.2 & 30.1 & 38.6 \\
\hline$>\$ 50,000$ & 11.8 & 34.3 & 25.0 & 43.1 \\
\hline \multicolumn{5}{|l|}{ Education level $(\%)^{c}$} \\
\hline Basic & 37.0 & 15.0 & 42.4 & 17.1 \\
\hline Intermediate & 30.1 & 51.1 & 26.8 & 39.0 \\
\hline Advanced & 32.9 & 33.9 & 30.8 & 43.9 \\
\hline \multicolumn{5}{|l|}{ Smoking status (\%) } \\
\hline Current & 25.5 & 24.9 & 38.0 & 24.6 \\
\hline Former & 17.2 & 25.1 & 34.0 & 47.3 \\
\hline Never & 57.3 & 50.0 & 27.9 & 28.1 \\
\hline \multicolumn{5}{|l|}{ Alcohol use (\%) } \\
\hline Current & 23.1 & 63.0 & 51.3 & 70.4 \\
\hline Former & 19.1 & 13.2 & 26.7 & 19.6 \\
\hline Never & 57.8 & 23.8 & 22.1 & 10.0 \\
\hline Leisure activity index ${ }^{d}$ & $2.3 \pm 0.7$ & $2.0 \pm 0.7$ & $2.2 \pm 0.7$ & $1.8 \pm 0.7$ \\
\hline \multicolumn{5}{|l|}{ LDL-C (mg/dl) (\%) } \\
\hline$\geq 160$ & 26.7 & 23.3 & 27.5 & 27.6 \\
\hline $130-159$ & 24.2 & 28.2 & 27.2 & 33.3 \\
\hline $100-129$ & 29.4 & 30.3 & 27.5 & 27.3 \\
\hline$<100$ & 19.7 & 18.2 & 17.8 & 11.8 \\
\hline
\end{tabular}

${ }^{a}$ Six census tract socioeconomic measures were combined into a composite index and categorized as race-specific tertiles (low, medium, high).

${ }^{b}$ Total combined family income for the past 12 months.

${ }^{c}$ Education level defined as highest grade completed in school: basic education ( $\leq$ high school); intermediate education (high school graduate or technical training); and advanced education ( $\geq$ some college).

${ }^{d}$ Sport during leisure time index used.

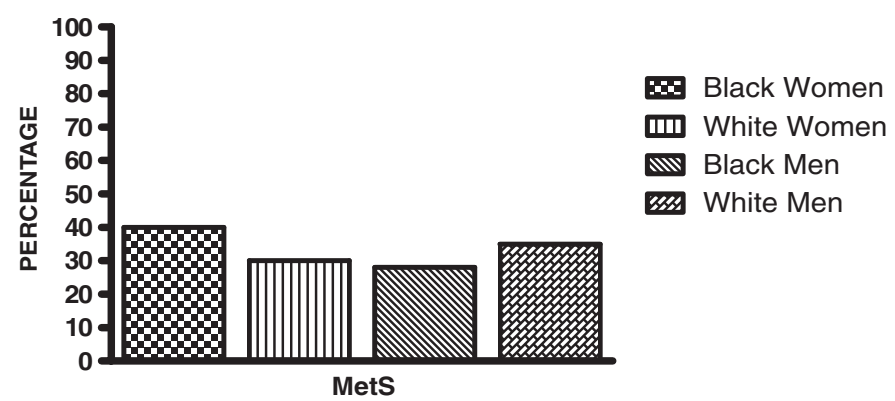

Figure 1. Most whites and black men were current drinkers of alcohol, whereas most black women were never drinkers. Age-adjusted prevalence of the metabolic syndrome by race and gender, Atherosclerosis Risk in Communities (ARIC) Study (1987-1989).

When iSES was included in models as a covariate, six household income categories were used (under $\$ 15,000, \$ 16,000$ to $\$ 24,999, \$ 25,000$ to $\$ 34,999, \$ 35,000$ to $\$ 49,999, \$ 50,000$ to $\$ 74,999$, and $\$ 75,000$ or more).

\section{RESULTS}

Table 1 presents selected characteristics of the study population by race and gender. When compared with whites, black men and women were disproportionately represented in the lower education and family income categories, as well as in poorer neighborhoods. Most black and white women were never smokers, whereas most white men were former smokers. In contrast, black men were approximately equally distributed across the smoking status categories. Whites had lower levels of physical activity than blacks. Age (mean between 53 and 55 years) and patterns of LDL-C (51\%-61\% had LDL-C $\geq 130 \mathrm{mg} / \mathrm{dL}$ ) were similar among black and white, men and women.

Figure 1 presents the data on the age-adjusted prevalence of the MetS for each race-gender group. The MetS was highest in black women $(40 \%)$, followed by white men $(35 \%)$, white women (30\%), and black men (28\%).

\section{SES and the MetS}

Table 2 presents the age-adjusted prevalence of the MetS by iSES and nSES for each race-gender group. Among whites, within gender groups, patterns were similar for iSES and nSES: Among women there were inverse gradients between SES and MetS and among men, although a gradient was not present, those in the highest SES group had a modestly lower prevalence of MetS. In contrast, among black men there was no clear association between nSES or iSES and the MetS, 
TABLE 2. The Age-Adjusted Prevalence of the Metabolic Syndrome and Neighborhood Index Score Income by Race and Gender, Atherosclerosis Risk in Communities (ARIC) Study (1987-1989) ${ }^{a}$

\begin{tabular}{|c|c|c|c|c|}
\hline$n$ & $\begin{array}{c}\text { Black Women } \\
1803\end{array}$ & $\begin{array}{l}\text { White Women } \\
\qquad 5244\end{array}$ & $\begin{array}{c}\text { Black Men } \\
1129\end{array}$ & $\begin{array}{c}\text { White Men } \\
\quad 4533\end{array}$ \\
\hline \multicolumn{5}{|l|}{ Family income ${ }^{b}$} \\
\hline$>\$ 50,000$ & $21(15-31)$ & $21(18-23)$ & $30(22-41)$ & $31(28-34)$ \\
\hline$\$ 25,000-\$ 49,999$ & $36(29-44)$ & $28(25-30)$ & $29(23-38)$ & $36(33-40)$ \\
\hline$<\$ 25,000$ & $45(40-50)$ & $35(32-39)$ & $30(24-37)$ & $35(30-40)$ \\
\hline \multicolumn{5}{|c|}{ Neighborhood Z-Score ${ }^{c}$} \\
\hline High & $36(32-42)$ & $23(20-24)$ & $28(25-32)$ & $33(30-35)$ \\
\hline Medium & $43(39-47)$ & $30(28-32)$ & $30(27-33)$ & $35(33-37)$ \\
\hline Low & $44(40-49)$ & $35(32-38)$ & $27(24-31)$ & $36(33-40)$ \\
\hline
\end{tabular}

${ }^{a}$ Data are percents (confidence interval).

${ }^{b}$ Total combined family income for the past 12 months.

${ }^{c}$ Six census tract socioeconomic measures were combined into a composite index and categorized as race-specific tertiles (low, medium, high).

whereas among black women there was an inverse association between SES and MetS and a lower prevalence of MetS among those in high nSES areas compared with those in middle and lower nSES areas. Results for education (not shown) are similar to those seen for family income.

We assessed for effect measure modification of the iSES and nSES associations with MetS by race and gender. There was evidence of race and gender differences in the association of iSES and nSES with MetS prevalence ( $p$ interaction $<.05$ for each). Thus, results are presented stratified by race and gender. Table 3 presents multivariable adjusted PRs and 95\% CIs for the association between iSES and nSES and the MetS by race and gender. Among black and white men, there was no association between iSES or nSES and the MetS. In contrast, among black and white women there were graded inverse associations between iSES and the MetS (black women: low versus high iSES ( $\mathrm{PR}=1.92,95 \% \mathrm{CI}, 1.41-2.61)$ and medium versus high iSES (PR $=1.56,95 \% \mathrm{CI}, 1.03-2.34)$; white women: low versus high iSES (PR $=1.36,95 \% \mathrm{CI}, 1.18-$ $1.58)$ and medium versus high iSES ( $\mathrm{PR}=1.20,95 \% \mathrm{CI}$, 1.05-1.37)). Among women, inverse associations of nSES with MetS were also observed. The magnitude of this association was weaker than the association observed for iSES in both black women (low versus high nSES $(\mathrm{PR}=1.20,95 \%$
CI, 1.04-1.40) and medium versus high nSES (PR $=1.25$, 95\% CI, 1.03-1.53)) and white women (low versus high nSES $(\mathrm{PR}=1.17,95 \% \mathrm{CI}, 1.00-1.37)$; and medium versus high nSES (PR $=1.14,95 \%$ CI, 1.00-1.30)). Results for education, not shown, were similar to those obtained when family income was used as a proxy for iSES.

We repeated analyses including all participants who had prevalent diabetes at baseline. Associations of both iSES and nSES with MetS remained similar (data not shown).

\section{DISCUSSION}

Our results are consistent with earlier studies, which report an inverse association between iSES and the MetS (21-36), although associations were largely limited to women. We also found evidence of a modest increased prevalence of MetS among those from medium and lower SES neighborhoods. This nSES effect, though not always significant, persisted after controlling for iSES and was evident in all race-gender groups except for white men. The association of neighborhood SES with MetS has not been well studied in the literature, although a pattern of weaker associations between nSES and insulin resistance in men compared with women has also been observed by Diez-Roux et al. (38). However, they found an inverse association between SES and the MetS in white men

TABLE 3. Adjusted $^{a}$ Prevalence Ratios and $95 \%$ Confidence Intervals of the Metabolic Syndrome by Income and Neighborhood Index Score by Race and Gender, Atherosclerosis Risk in Communities (ARIC) Study (1987-1989)

\begin{tabular}{|c|c|c|c|c|}
\hline$n$ & $\begin{array}{c}\text { Black Women } \\
1803\end{array}$ & $\begin{array}{l}\text { White Women } \\
5244\end{array}$ & $\begin{array}{c}\text { Black Men } \\
1129\end{array}$ & $\begin{array}{l}\text { White Men } \\
\quad 4533\end{array}$ \\
\hline \multicolumn{5}{|l|}{ Family income $e^{b}$} \\
\hline$>\$ 50,000$ & 1.00 & 1.00 & 1.00 & 1.00 \\
\hline$\$ 25,000-\$ 49,999$ & $1.56(1.03-2.34)$ & $1.20(1.05-1.37)$ & $0.97(0.63-1.52)$ & $1.14(1.03-1.26)$ \\
\hline$<\$ 25,000$ & $1.92(1.41-2.61)$ & $1.36(1.18-1.58)$ & $0.98(0.68-1.41)$ & $1.04(0.90-1.20)$ \\
\hline \multicolumn{5}{|c|}{ Neighborhood Z-Score ${ }^{c}$} \\
\hline High & 1.00 & 1.00 & 1.00 & 1.00 \\
\hline Medium & $1.25(1.03-1.53)$ & $1.14(1.00-1.30)$ & $1.28(1.01-1.62)$ & $0.99(0.88-1.12)$ \\
\hline Low & $1.20(1.04-1.40)$ & $1.17(1.00-1.37)$ & $1.16(0.89-1.50)$ & $0.98(0.84-1.15)$ \\
\hline
\end{tabular}

\footnotetext{
${ }^{a}$ Model adjusted for age, smoking status, alcohol use, physical activity, LDL-C, ARIC center and jointly considered iSES and nSES.

${ }^{b}$ Total combined family income for the past 12 months.

${ }^{c}$ Six census tract socioeconomic measures were combined into a composite index and categorized as race-specific tertiles (low, medium, high).
} 
and in black men in the highest categories of income and education, whereas we did not observe significant associations for either black or white men.

Different proxy measures of SES (e.g., education, income) are known to vary in how accurately they reflect the socioeconomic circumstances of different sociodemographic groups $(55,56)$. Thus, it is possible that the observed gender differences reflect errors in measurement of SES. However, our findings were consistent across measures and our neighborhood measure incorporated a range of characteristics previously reported to contribute to the social and economic context of area of residence (50), minimizing our concerns that the observed gender differences were merely artifactual.

There are several mechanisms by which SES may differentially affect the MetS in women and men. It has been suggested that abdominal obesity and defects in insulin sensitivity are the two main risk factors for the MetS (57-59). In addition to being inversely correlated with insulin sensitivity (60), abdominal obesity often leads to insulin resistance $(57,59)$. Various metabolic pathways have been proposed to link both insulin resistance and increased waist circumference to the MetS (61). Thus, it is possible that the inverse SESMetS associations seen in women are in part due to a higher prevalence of large waist in women than in men. In support of this hypothesis, other studies have observed gender differences in SES-obesity/body mass index associations $(11,12)$. However, when we examined each component of the MetS individually (data not shown), we did not observe stronger associations of SES with large-waist. Furthermore, statistically significant inverse associations between the prevalence of large-waist and iSES were limited to white women. Second, the association between low SES and distress (62) and depression $(63,64)$ is robust in the literature. It has been postulated that persons with low SES have less personal resources, such as locus of control, coping styles, social support, and experience more stressful life events, than persons with higher SES and are thus more vulnerable to the negative effects of stress on health $(65,66)$. Furthermore, in the population as a whole, women are more likely than men to experience depression $(67,68)$. Stress and depression, via the autonomic nervous system and dysregulation of the hypothalamic-pituitary-adrenocortical axis $(62,69)$, are plausible risk factors for the MetS (70,71). In a nationally representative sample of US adults, a history of depression was independently associated with the prevalence of MetS in women but not men (72). Thus, it is plausible that the observed gender differences in the association of SES with MetS were at least in part due to different psychological responses to adverse socioeconomic circumstances. However, these psychosocial measures were not ascertained in the ARIC baseline examination, and thus we could not evaluate their potential role in the current study.

Gender and race differences in the association between SES and the MetS have been reported in the literature $(23,38)$. A recent report based on a nationally representative sample found that among women, inverse associations of education with MetS were stronger among white women than black women and among men, modest inverse associations of education with MetS were observed in white men (23). Furthermore, an earlier report based on young adults found an inverse association between nSES and the insulin resistance syndrome in whites, and black women whereas in black men the inverse association with nSES was limited only to those persons with more favorable individual social and economic circumstances (high education and income) (38). In our study, blacks disproportionately resided in less affluent neighborhoods and there were relatively proportionately few blacks represented in the high nSES tertile neighborhoods. The limited overlap in the distribution of black and white participants by nSES and the small numbers of black participants in higher SES neighborhoods in the ARIC study communities necessitated the use of race-specific tertiles of neighborhood summary scores. Given this approach, direct comparisons between the magnitude of the nSES-MetS associations in blacks and whites may not be valid.

Interpretations of differences in the relation between SES and MetS by race are further complicated by the confounding between race and geographic region. Both Washington County, MD and Minneapolis, MN study samples consisted solely of white participants, and whereas the Forsyth County, NC study sample consisted of both black and white participants, Jackson, MS, with the lowest level of neighborhood socioeconomic characteristics of the study communities, consisted of black participants only.

This study had several strengths. First, it extends findings from previous US reports that showed varying race and gender associations between SES and the MetS. Second, it is based on a large biracial cohort with standardized risk factor assessment of men and women from diverse communities in the US population. Furthermore, we included a variety of SES measures that allowed us to assess the impact of unfavorable individual and contextual social and economic exposures on the MetS.

The current study also has limitations that should be acknowledged. The black population included only two southern US communities that may not be typical of blacks in the United States. However, it is reassuring that within gender groups, the pattern of our findings tended to be consistent across black and white participants. Our study was crosssectional and thus, we cannot infer causality. As mentioned earlier, psychosocial measures, such as stress and depression, are potentially important mediators that deserve attention in future analyses of the relationship between SES and MetS.

There is debate in the literature about the appropriate geographical level at which to define neighborhood. Further, there is some evidence of variations in the association of neighborhood characteristics with health outcomes when boundaries are changed (the modifiable area unit problem) (73). In the current study, we used census tracts to represent neighborhoods. Census tracts are relatively stable units designed to be homogeneous with regards to population and economic and living conditions (74). Further, empirical associations of neighborhood socioeconomic characteristics defined at the level of the census tract with health outcomes have 
been found to be virtually the same as when smaller census block groups were used to define neighborhood $(41,75,76)$. Finally, fixed cutpoints were used to classify each component of the MetS. Use of fixed cutpoints could result in misclassification of the MetS; further, it is also possible that the appropriateness of these cutpoints may vary across race and gender groups.

Although race and gender differences in the prevalence of MetS are well described in the literature, the intersections between race, gender, and the joint contribution of iSES and nSES and MetS have received little attention. In the current study, among both black and white participants, iSES and nSES were inversely associated with an increased prevalence of MetS among women but not men. Further studies aimed at identifying mechanisms, which explain the variations in the impact of SES on MetS are warranted and may provide insight into avenues for the prevention of the MetS and its chronic disease sequelae.

The authors thank the staff and participants of the ARIC study for their important contributions.

\section{REFERENCES}

1. Executive summary of the third report of the National Cholesterol Education Program (NCEP) Expert Panel on detection, evaluation, and treatment of high blood cholesterol in adults (Adult Treatment Panel III). JAMA 2001;285:2486-97.

2. Zimmet P, Magliano D, Matsuzawa Y, Alberti G, Shaw J. The metabolic syndrome: a global public health problem and a new definition. J Atheroscler Thromb 2005;12:295-300.

3. Ford ES, Giles WH, Dietz WH. Prevalence of the metabolic syndrome among US adults: findings from the third National Health and Nutrition Examination Survey. JAMA 2002;287:356-9.

4. Laaksonen DE, Lakka HM, Niskanen LK, Kaplan GA, Salonen JT, Lakka TA. Metabolic syndrome and development of diabetes mellitus: application and validation of recently suggested definitions of the metabolic syndrome in a prospective cohort study. Am J Epidemiol 2002;156: $1070-7$.

5. Lorenzo C, Williams K, Hunt KJ, Haffner SM. The National Cholesterol Education Program-Adult Treatment Panel III, International Diabetes Federation, and World Health Organization definitions of the metabolic syndrome as predictors of incident cardiovascular disease and diabetes. Diabetes Care 2007;30:8-13.

6. McNeill AM, Rosamond WD, Girman CJ, Heiss G, Golden SH, Duncan BB, East HE, Ballantyne C. Prevalence of coronary heart disease and carotid arterial thickening in patients with the metabolic syndrome (The ARIC Study). Am J Cardiol 2004;94:1249-54.

7. McNeill AM, Rosamond WD, Girman CJ, Golden SH, Schmidt MI, East HE, Ballantyne CM, Heiss G. The metabolic syndrome and 11-year risk of incident cardiovascular disease in the atherosclerosis risk in communities study. Diabetes Care 2005;28:385-90.

8. Malik S, Wong ND, Franklin SS, Kamath TV, L'Italien GJ, Pio JR, Williams GR. Impact of the metabolic syndrome on mortality from coronary heart disease, cardiovascular disease, and all causes in United States adults. Circulation 2004;110:1245-50.

9. Ninomiya JK, L'Italien G, Criqui MH, Whyte JL, Gamst A, Chen RS. Association of the metabolic syndrome with history of myocardial infarction and stroke in the Third National Health and Nutrition Examination Survey. Circulation 2004;109:42-6.

10. Wamala SP, Wolk A, Orth-Gomer K. Determinants of obesity in relation to socioeconomic status among middle-aged Swedish women. Prev Med 1997;26:734-44.

11. Sobal J, Stunkard AJ. Socioeconomic status and obesity: a review of the literature. Psychol Bull 1989;105:260-75.

12. Dekkers JC, Podolsky RH, Treiber FA, Barbeau P, Gutin B, Snieder H. Development of general and central obesity from childhood into early adulthood in African American and European American males and fe- males with a family history of cardiovascular disease. Am J Clin Nutr 2004;79:661-8.

13. Cubbin C, Sundquist K, Ahlen H, Johansson SE, Winkleby MA, Sundquist J. Neighborhood deprivation and cardiovascular disease risk factors: protective and harmful effects. Scand J Public Health 2006;34: $228-37$.

14. Choi BC, Shi F. Risk factors for diabetes mellitus by age and sex: results of the National Population Health Survey. Diabetologia 2001;44: 1221-31.

15. Connolly V, Unwin N, Sherriff P, Bilous R, Kelly W. Diabetes prevalence and socioeconomic status: a population based study showing increased prevalence of type 2 diabetes mellitus in deprived areas. J Epidemiol Community Health 2000;54:173-7.

16. Tang M, Chen Y, Krewski D. Gender-related differences in the association between socioeconomic status and self-reported diabetes. Int $\mathrm{J}$ Epidemiol 2003;32:381-5.

17. Colhoun HM, Hemingway H, Poulter NR. Socioeconomic status and blood pressure: an overview analysis. J Hum Hypertens 1998;12:91-110.

18. Matthews KA, Kiefe CI, Lewis CE, Liu K, Sidney S, Yunis C. Socioeconomic trajectories and incident hypertension in a biracial cohort of young adults. Hypertension 2002;39:772-6.

19. Freedman DS, Strogatz DS, Williamson DF, Aubert RE. Education, race, and high-density lipoprotein cholesterol among US adults. Am J Public Health 1992;82:999-1006.

20. Watkins LO, Neaton JD, Kuller LH. Racial differences in high-density lipoprotein cholesterol and coronary heart disease incidence in the usualcare group of the Multiple Risk Factor Intervention Trial. Am J Cardiol 1986;57:538-45.

21. Carnethon MR, Loria CM, Hill JO, Sidney S, Savage PJ, Liu K. Risk factors for the metabolic syndrome: the Coronary Artery Risk Development in Young Adults (CARDIA) study, 1985-2001. Diabetes Care 2004;27:2707-15.

22. Loucks EB, Magnusson KT, Cook S, Rehkopf DH, Ford ES, Berkman LF. Socioeconomic position and the metabolic syndrome in early, middle, and late life: evidence from NHANES 1999-2002. Ann Epidemiol 2007;17:782-90.

23. Loucks EB, Rehkopf DH, Thurston RC, Kawachi I. Socioeconomic disparities in metabolic syndrome differ by gender: evidence from NHANES III. Ann Epidemiol 2007;17:19-26.

24. Lucove JC, Kaufman JS, James SA. Association between adult and childhood socioeconomic status and prevalence of the metabolic syndrome in African Americans: the Pitt County Study. Am J Public Health 2007;97:234-6.

25. Park YW, Zhu S, Palaniappan L, Heshka S, Carnethon MR, Heymsfield SB. The metabolic syndrome: prevalence and associated risk factor findings in the US population from the Third National Health and Nutrition Examination Survey, 1988-1994. Arch Intern Med 2003;163: 427-36.

26. Salsberry PJ, Corwin E, Reagan PB. A complex web of risks for metabolic syndrome: race/ethnicity, economics, and gender. Am J Prev Med 2007;33:114-20.

27. Brunner EJ, Marmot MG, Nanchahal K, Shipley MJ, Stansfeld SA, Juneja M, Alberti KG. Social inequality in coronary risk: central obesity and the metabolic syndrome. Evidence from the Whitehall II study. Diabetologia 1997;40:1341-9.

28. Dallongeville J, Cottel D, Ferrieres J, Arveiler D, Bingham A, Ruidavets JB, Haas B, Ducimetiere P, Amouyel P. Household income is associated with the risk of metabolic syndrome in a sex-specific manner. Diabetes Care 2005;28:409-15.

29. Kim MH, Kim MK, Choi BY, Shin YJ. Educational disparities in the metabolic syndrome in a rapidly changing society - the case of South Korea. Int J Epidemiol 2005;34:1266-73.

30. Langenberg C, Kuh D, Wadsworth ME, Brunner E, Hardy R. Social circumstances and education: life course origins of social inequalities in metabolic risk in a prospective national birth cohort. Am J Public Health 2006;96:2216-21

31. Park MJ, Yun KE, Lee GE, Cho HJ, Park HS. A cross-sectional study of socioeconomic status and the metabolic syndrome in Korean adults. Ann Epidemiol 2007;17:320-6.

32. Perel P, Langenberg C, Ferrie J, Moser K, Brunner E, Marmot M. Household wealth and the metabolic syndrome in the Whitehall II study. Diabetes Care 2006;29:2694-700.

33. Santos AC, Ebrahim S, Barros H. Gender, socioeconomic status and 
metabolic syndrome in middle-aged and old adults. BMC Public Health 2008;8:62.

34. Schooling CM, Jiang CQ, Lam TH, Zhang WS, Cheng KK, Leung GM. Life-course origins of social inequalities in metabolic risk in the population of a developing country. Am J Epidemiol 2008;167:419-28.

35. Silventoinen K, Pankow J, Jousilahti P, Hu G, Tuomilehto J. Educational inequalities in the metabolic syndrome and coronary heart disease among middle-aged men and women. Int J Epidemiol 2005;34:327-34.

36. Wamala SP, Lynch J, Horsten M, Mittleman MA, Schenck-Gustafsson K, Orth-Gomer K. Education and the metabolic syndrome in women. Diabetes Care 1999;22:1999-2003.

37. Borrell LN, Diez Roux AV, Rose K, Catellier D, Clark BL. Neighbourhood characteristics and mortality in the Atherosclerosis Risk in Communities Study. Int J Epidemiol 2004;33:398-407.

38. Diez Roux AV, Jacobs DR, Kiefe CI. Neighborhood characteristics and components of the insulin resistance syndrome in young adults: the coronary artery risk development in young adults (CARDIA) study. Diabetes Care 2002;25:1976-82.

39. Diez Roux AV, Merkin SS, Arnett D, Chambless L, Massing M, Nieto FJ, Sorlie P, Szklo M, Tyroler HA, Watson RL. Neighborhood of residence and incidence of coronary heart disease. N Engl J Med 2001;345: 99-106.

40. Nordstrom CK, Diez Roux AV, Jackson SA, Gardin JM. The association of personal and neighborhood socioeconomic indicators with subclinical cardiovascular disease in an elderly cohort. The cardiovascular health study. Soc Sci Med 2004;59:2139-47.

41. Diez Roux AV, Merkin SS, Hannan P, Jacobs DR, Kiefe CI. Area characteristics, individual-level socioeconomic indicators, and smoking in young adults: the coronary artery disease risk development in young adults study. Am J Epidemiol 2003;157:315-26.

42. Hill TD, Ross CE, Angel RJ. Neighborhood disorder, psychophysiological distress, and health. J Health Soc Behav 2005;46:170-86.

43. Ross CE, Mirowsky J. Neighborhood disadvantage, disorder, and health. J Health Soc Behav 2001;42:258-76.

44. Moore LV, Diez Roux AV. Associations of neighborhood characteristics with the location and type of food stores. Am J Public Health 2006;96: $325-31$.

45. Stafford M, Marmot M. Neighbourhood deprivation and health: does it affect us all equally? Int J Epidemiol 2003;32:357-66.

46. Jackson R, Chambless LE, Yang K, Byrne T, Watson R, Folsom A, Shahar E, Kalsbeek W. Differences between respondents and nonrespondents in a multicenter community-based study vary by gender ethnicity. The Atherosclerosis Risk in Communities (ARIC) Study Investigators. J Clin Epidemiol 1996;49:1441-6.

47. The Atherosclerosis Risk in Communities (ARIC) Study: design and objectives. The ARIC investigators. Am J Epidemiol 1989;129:687-702.

48. Lifecourse Socioeconomic Status, Social Context and Cardiovascular Disease (LC-SES) Study: manual of procedures. http://www.lifecourseepi. info/lifecourse2/forms/MOP3904_web.pdf. Accessed August 4, 2008.

49. Whitsel EA, Quibrera PM, Smith RL, Catellier DJ, Liao D, Henley AC, Heiss G. Accuracy of commercial geocoding: assessment and implications. Epidemiol Perspect Innov 2006;3:8.

50. Diez-Roux AV, Kiefe CI, Jacobs DR Jr, Haan M, Jackson SA, Nieto FJ, Paton CC, Schulz R. Area characteristics and individual-level socioeconomic position indicators in three population-based epidemiologic studies. Ann Epidemiol 2001;11:395-405.

51. Kaufman JS, Cooper RS. Race in epidemiology: new tools, old problems. Ann Epidemiol 2008;18:119-23.

52. Baecke JA, Burema J, Frijters JE. A short questionnaire for the measurement of habitual physical activity in epidemiological studies. Am J Clin Nutr 1982;36:936-42.

53. McNutt LA, Wu C, Xue X, Hafner JP. Estimating the relative risk in cohort studies and clinical trials of common outcomes. Am J Epidemiol 2003;157:940-3.
54. Allison P. Logistic regression using the SAS system. Cary, NC: SAS; 1999.

55. Krieger N, Williams DR, Moss NE. Measuring social class in US public health research: concepts, methodologies, and guidelines. Annu Rev Public Health 1997;18:341-78.

56. Liberatos P, Link BG, Kelsey JL. The measurement of social class in epidemiology. Epidemiol Rev 1988;10:87-121.

57. Kahn R. Metabolic syndrome: is it a syndrome? Does it matter? Circulation 2007;115:1806-10; discussion 1811 .

58. Meigs JB. The metabolic syndrome. BMJ 2003;327:61-2.

59. Grundy SM. Metabolic syndrome: connecting and reconciling cardiovascular and diabetes worlds. J Am Coll Cardiol 2006;47:1093-100.

60. Groop L, Orho-Melander M. The dysmetabolic syndrome. J Intern Med 2001;250:105-20.

61. Eckel RH, Grundy SM, Zimmet PZ. The metabolic syndrome. Lancet 2005;365:1415-28.

62. Cohen S, Doyle WJ, Baum A. Socioeconomic status is associated with stress hormones. Psychosom Med 2006;68:414-20.

63. Lorant V, Deliege D, Eaton W, Robert A, Philippot P, Ansseau M. Socioeconomic inequalities in depression: a meta-analysis. Am J Epidemiol 2003;157:98-112.

64. Koster A, Bosma H, Kempen GI, Penninx BW, Beekman AT, Deeg DJ, van Eijk JT. Socioeconomic differences in incident depression in older adults: the role of psychosocial factors, physical health status, and behavioral factors. J Psychosom Res 2006;61:619-27.

65. Taylor SE, Seeman TE. Psychosocial resources and the SES-health relationship. Ann N Y Acad Sci 1999;896:210-25.

66. Bosma H, Van Jaarsveld CH, Tuinstra J, Sanderman R, Ranchor AV, Van Eijk JT, Kempen GI. Low control beliefs, classical coronary risk factors, and socioeconomic differences in heart disease in older persons. Soc Sci Med 2005;60:737-45.

67. Weissman MM, Bland R, Joyce PR, Newman S, Wells JE, Wittchen HU. Sex differences in rates of depression: cross-national perspectives. J Affect Disord 1993;29:77-84.

68. Weissman MM, Bland RC, Canino GJ, Faravelli C, Greenwald S, Hwu HG, Joyce PR, Karam EG, Lee CK, Lellouch J, Lepine JP, Newman SC, Rubio-Stipec M, Wells JE, Wickramaratne PJ, Wittchen H, Yeh EK. Cross-national epidemiology of major depression and bipolar disorder. JAMA 1996;276:293-9.

69. McEwen BS. Central effects of stress hormones in health and disease: understanding the protective and damaging effects of stress and stress mediators. Eur J Pharmacol 2008;583:174-85.

70. Chandola T, Brunner E, Marmot M. Chronic stress at work and the metabolic syndrome: prospective study. BMJ 2006;332:521-5.

71. Bjorntorp P. Do stress reactions cause abdominal obesity and comorbidities? Obes Rev 2001;2:73-86.

72. Kinder LS, Carnethon MR, Palaniappan LP, King AC, Fortmann SP. Depression and the metabolic syndrome in young adults: findings from the Third National Health and Nutrition Examination Survey. Psychosom Med 2004;66:316-22.

73. Flowerdew R, Manley DJ, Sabel CE. Neighbourhood effects on health: does it matter where you draw the boundaries? Soc Sci Med 2008;66: $1241-55$.

74. US Census Bureau. Appendix A: Census 2000 geographic terms and concepts. Available at: http://www.census.gov/geo/www/tiger/ glossry2.pdf. Accessed July 29, 2008.

75. Lovasi GS, Moudon AV, Smith NL, Lumley T, Larson EB, Sohn DW, Siscovick DS, Psaty BM. Evaluating options for measurement of neighborhood socioeconomic context: evidence from a myocardial infarction case-control study. Health Place 2007;14:453-67.

76. Krieger N, Chen JT, Waterman PD, Soobader MJ, Subramanian SV, Carson R. Geocoding and monitoring of US socioeconomic inequalities in mortality and cancer incidence: does the choice of area-based measure and geographic level matter? The Public Health Disparities Geocoding Project. Am J Epidemiol 2002;156:471-82. 